\title{
Comparison of the Electron Localization Function and Deformation Electron Density Maps for Selected Earth Materials
}

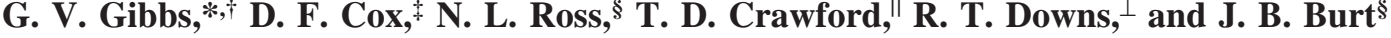 \\ Departments of Geosciences, Materials Science and Engineering, and Mathematics, Virginia Tech, \\ Blacksburg, Virginia 24061, Department of Chemical Engineering, Virginia Tech, Blacksburg, Virginia 24061, \\ Department of Geosciences, Virginia Tech, Blacksburg, Virginia 24061, Department of Chemistry, Virginia \\ Tech, Blacksburg, Virginia 24061, and Department of Geosciences, University of Arizona, \\ Tucson, Arizona 85721
}

Received: May 20, 2005; In Final Form: August 17, 2005

\begin{abstract}
The electron localization function (ELF) and experimental and theoretical deformation electron density maps are compared for several earth materials and one representative molecule. The number and arrangement of the localized one-electron probability density domains generated in a mapping of the ELF correspond to the number and arrangement of the localized electron density domains generated in a mapping of the deformation electron density distribution, a correspondence that suggests that the two fields are homeomorphically related. As a homeomorphic relationship has been established previously between the Laplacian of the electron density distribution and the ELF, the relationship suggests that the deformation electron density distribution is also homeomorphically related to the Laplacian of the distribution.
\end{abstract}

\section{Introduction}

In a mapping of the electron localization function, $\eta(r)$, and the Laplacian, $L(r)=-\nabla^{2} \rho(r)$, of the electron density distribution for a variety of geometry optimized hydride and fluoride molecules, Bader et al. ${ }^{1}$ found that level line contour and isosurface maps generated for the molecules show a correspondence in the number and arrangement of the locally concentrated and localized domains of electron density, evidence that the $L(r)$ and $\eta(r)$ distributions are homeomorphically related. However, $L(r)$ was suggested to be superior to $\eta(r)$ in the prediction of the positions and the magnitudes of the domains. It is noted that $L(r)$ is an empirical function that is grounded on the basis of its close connection with the well-known valenceshell electron-pair repulsion (VSEPR) model of molecular geometry. ${ }^{2}$

In a recent generation of $\eta(r)$ maps for a relatively large number of earth materials, ${ }^{3}$ well-defined isosurface domains of probability density were displayed along the bond vectors and in the lone-pair regions similar to that assumed in the VSEPR electron-pair domain model. As contour and isosurface $\Delta \rho(r)$ maps observed for several of the earth materials ${ }^{4-6}$ display features that are similar to those displayed by the electron localization function, $\eta(r)$, isosurface maps were generated in this study for several earth materials and a representative silicate molecule. A comparison of the maps is made to gauge the extent to which the two types of maps agree and to establish whether a connection exists between the topography of the domains of localized electron density ascribed to electron pairs. We will present evidence that suggests that a correspondence exists between the location and the arrangement of the domains

\footnotetext{
$\dagger$ Departments of Geosciences, Materials Science and Engineering, and Mathematics, Virginia Tech.

$\doteqdot$ Department of Chemical Engineering, Virginia Tech.

$\S$ Department of Geosciences, Virginia Tech.

" Department of Chemistry, Virginia Tech.

$\perp$ University of Arizona.
}

displayed by $\eta(r)$ and deformation electron density, $\Delta \rho$, maps. The correspondence will be established in a comparative study of the $\eta(r)$ and $\Delta \rho(r)$ distributions generated in this study.

\section{Geometry Optimization and the Electron Localization Function}

The structures of the hexahydroxydisiloxane molecule and the earth materials considered in this study were geometry optimized with VASP, a periodic plane wave density functional code written by Kresse and Hafner ${ }^{7,8}$ and Kresse and Furthmüller. ${ }^{9}$ The code uses ultrasoft pseudopotentials ${ }^{10}$ and the local density approximation (LDA) to model the contribution of the exchange correlation to the total energy and the valence electron density distribution. The kinetic energy cutoff and the density of the Monkhorst-Pack ${ }^{11} k$-point mesh were chosen to be of sufficient magnitude to ensure convergence of the energy and the generation of a minimum energy structure. The geometry optimization was completed with quasi-zero pressure minimum energy cell dimensions, determined for the structure within LDA formalisms. The coordinates of the nonequivalent atoms were each varied within the constraints of the observed space group type for each earth material until the forces on each atom were negligibly small and close to zero (less than $0.03 \mathrm{eV} \AA^{-1}$ ). By and large, the experimental bond lengths and angles were found to be in close agreement with the optimized values, agreeing typically within $\sim 1 \%$, on average. $\eta(r)$ for the bonded interactions was generated for each geometry optimized structure using the VASP software. Isosurface maps were generated with a modified version of the desktop 3D visualization software of Terriberry et al. ${ }^{12}$ The modified version allows the construction of dual $\eta(r)$ isosurfaces with arbitrarily chosen $\eta(r)$ values and associated colors. For this study, dual $\eta(r)$ values of 0.83 (gray color coded isosurface) and 0.85 (pink color coded isosurface) were used in the generation of the dual isosurfaces for the 

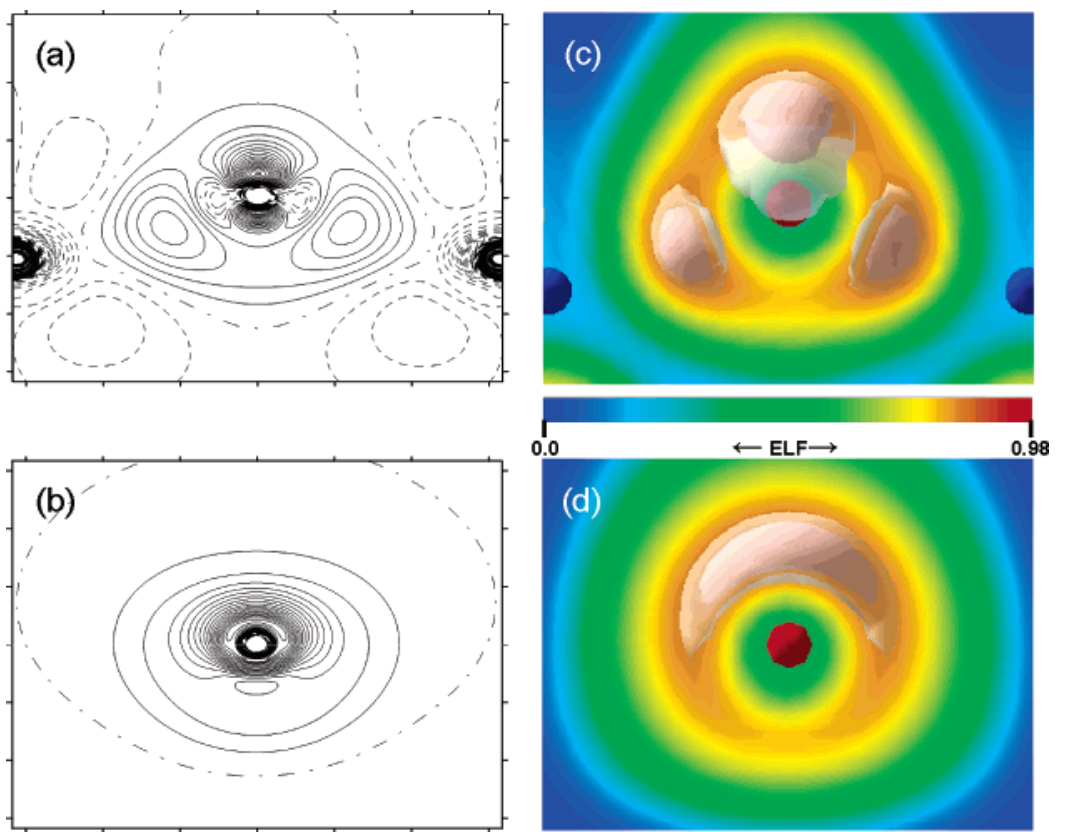

Figure 1. Theoretical deformation $\Delta \rho(r)$ level line contour maps (a and b) and electron localization function $\eta(r)$ dual isosurface maps (c and d) for the $(\mathrm{HO})_{3} \mathrm{SiOSi}(\mathrm{OH})_{3}$ molecule. The maps displayed in parts a and c were constructed for a plane containing the SiOSi angle, and those displayed in parts $b$ and $d$ were constructed for a perpendicular plane that bisects the angle. The contour interval displayed in parts $a$ and $b$ is 0.05 e $\AA^{-3}$, the dashed lines represent negative contours, the solid lines represent positive contours, and the dash-dotted line represents the zero level line contour. The gray $\eta(r)$ isosurface displayed in parts $\mathrm{c}$ and $\mathrm{d}$ represents an $\eta$ value of 0.83 , whereas the pink one represents an $\eta(r)$ value of 0.85. The color contour code is defined in the bar. The red spheres in parts $\mathrm{c}$ and $\mathrm{d}$ represent oxygen atoms, and the blue ones in part $\mathrm{c}$ represent silicon atoms.

figures. Color $\eta(r)$ contour maps were also constructed; the color code is defined in a bar displayed in each figure displaying color contours.

For the vast majority of bonded systems of chemical interest, Burdett and McCormick ${ }^{13}$ have demonstrated that the dominant term in $\eta(r)$ is $\rho(r)_{\sigma}^{5 / 3} / \sum_{i}\left|\nabla \psi(r)_{i, \sigma}\right|^{2}$, which appears in the denominator of the $\eta(r)$ definition, ${ }^{14}$ where $\rho(r)_{\sigma}=\left|\psi(r)_{i, \sigma}\right|^{2}$ is the one-electron orbital probability density and $\sum_{i}\left|\nabla \psi(r)_{i, \sigma}\right|^{2}$ is the kinetic energy density at $r$. Qualitatively, the value of the function is expected to be relatively large in those regions where the one-electron probability density distribution is relatively large (regions of overlapping atomic orbitals and well localized electrons) and where populated nodal planes are absent or few in number. On the other hand, the function is expected to be relatively small in those regions between atoms where populated nodal planes are commonly large in number (where the gradient of $\psi(r)$ is relatively large) or the electron probability density is small or both. Becke and Edgecombe ${ }^{14}$ found it convenient in their derivation to restrict the range of the unitless $\eta(r)$ to lie between 0 and 1 with a value of 1 corresponding to perfect localization and a value of 0.5 corresponding to a complete delocalization of the one-electron probability density. For earth materials, values less than 0.5 are typically adopted in regions between domains of localized probability density with the function approaching 0.0 in the vicinity of metal atoms where there is a paucity of localized valence one-electron probability density. Further, like the $\Delta \rho(r)$ electron density distribution for each earth material, the $\eta(r)$ one-electron probability density distribution likewise obeys the observed space group symmetry type of the material. The spin states of the electrons were ignored in the VASP calculations and in the generation of $\eta(r)$ maps.

\section{Comparison of the Electron Localization Function (ELF) and Deformation Electron Density Distributions}

Hexahydroxydisiloxane, $(\mathrm{HO})_{3} \mathrm{SiOSi}(\mathrm{OH})_{3}$, Molecule. $\eta(r)$ isosurface and $\Delta \rho$ level line contour maps generated for a geometry optimized structure of the molecule display a similar number and arrangement of the local maxima in the bonded and nonbonded regions. ${ }^{15}$ The $\Delta \rho$ map for the plane defined by the SiOSi angle displays level lines that extend into the interior of the angle and envelop three maxima, two in the bonded regions, one each along the two $\mathrm{SiO}$ bond vectors, and the third in the nonbonded region on the reflex side of the angle near the $\mathrm{O}$ atom (Figure 1a). A second map, generated for the perpendicular plane that bisects the angle, displays a torus shaped array of level lines that surrounds the $\mathrm{O}$ atom and displays a banana shaped closed contour on the reflex side of the angle (Figure 1b). The $\eta(r)$ dual isosurface map generated for the SiOSi angle displays hemispherical isosurfaces along each of the $\mathrm{SiO}$ bond vectors and a banana shaped isosurface in the nonbonded region disposed perpendicular to the angle (Figure 1c). It also displays hemispherical isosurfaces along each of the nonbridging $\mathrm{Si}(\mathrm{OH})$ bond vectors and banana shaped isosurfaces oriented perpendicular to the $\mathrm{SiOH}$ angles (see Figure 4 in ref 15). Each of the banana shaped isosurfaces is irreducible and as such encloses a single maximum. The $\eta(r)$ color contour and dual isosurface maps generated for the SiOSi interaction and for the bisecting plane are displayed in parts $\mathrm{c}$ and $\mathrm{d}$ of Figure 1, respectively. Consistent with topography of the level line contour map for the SiOSi bonded interaction (Figure 1a), the $\mathrm{O}$ atom is surrounded by a triangular array of color contours which increase from light green to orange as the $\eta(r)$ value increases from about 0.5 to 0.87 (Figure 1c). Also, the color contours in Figure 1d surround the $\mathrm{O}$ atom in a torus as displayed in the $\Delta \rho(r)$ map in Figure 1b. The $\Delta \rho$ maxima along the $\mathrm{SiO}$ vectors are located closer to the $\mathrm{O}$ atom $(\sim 0.65$ $\AA)$ than the $\eta(r)$ maxima $(\sim 0.70 \AA)$. Likewise, the $\Delta \rho$ maxima in the nonbonded region are closer to the $\mathrm{O}(\sim 0.20 \AA)$ than the $\eta(r)$ maxima $(\sim 0.50 \AA)$. Although the maximum in the lonepair region (Figure $1 \mathrm{~b}$ ) is located much closer to the $\mathrm{O}$ atom than the maximum of the banana shaped isosurface, the features displayed by the two sets of maps are similar in number and 


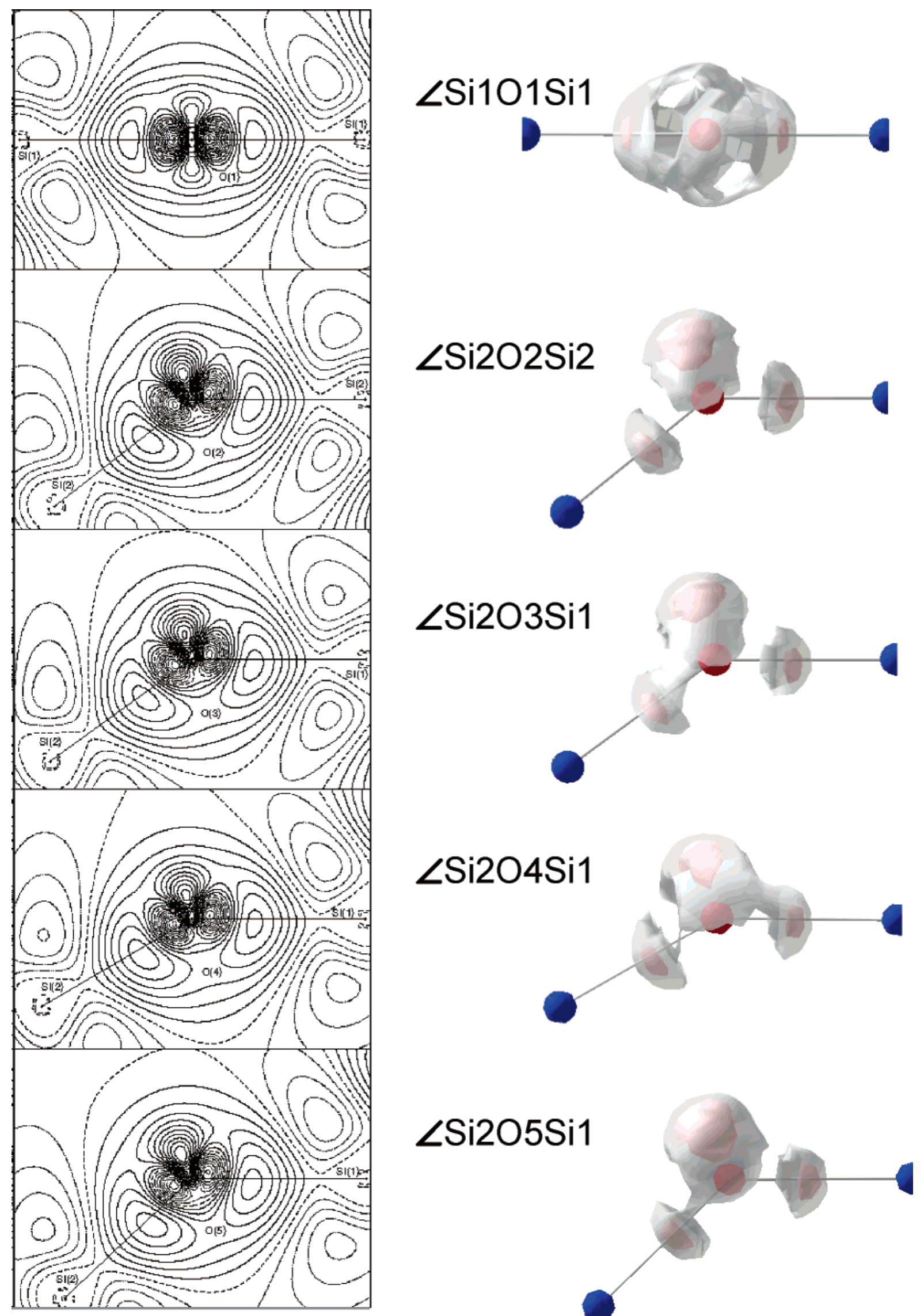

Figure 2. Static model $\Delta \rho(r)$ level line contour maps (left column 1) and $\eta(r)$ isosurface maps (right column 2) for the $\mathrm{Si} 1 \mathrm{O} 1 \mathrm{Si} 1, \mathrm{Si} 2 \mathrm{O} 2 \mathrm{Si} 2$, Si2O3Si1, Si2O4Si1, and Si2O5Sil bonded interactions in coesite, running top to bottom. The contour interval is 0.05 e $\AA^{-3}$, the solid lines represent positive contours, the dotted lines represent negative ones, and the dashed lines represent zero contour. The Si1O1Si1, Si2O2Si2, Si2O3Si1, $\mathrm{Si} 2 \mathrm{O} 4 \mathrm{Si}$, and $\mathrm{Si} 2 \mathrm{O} 5 \mathrm{Si} 1$ angles are $180,142.0,144.2,149.7$, and $136.7^{\circ}$, respectively. The information defining the isosurfaces is given in the legend for Figure 1. The red spheres represent oxygen atoms, and the blue ones represent silicon atoms. 
arrangement. The heights of the bonded and nonbonded maxima displayed in the $\eta(r)$ isosurface maps are similar, unlike the height of the maximum displayed in the nonbonded region of the $\Delta \rho(r)$ map, a maximum that is substantially higher than that displayed in the bonded region along each $\mathrm{SiO}$ bond vector. In general, this is not surprising given that the deformation density is defined as the difference between the total electron density and the density calculated with a reference model based on unbiased positional and thermal parameters ${ }^{16}$ and usually represents the difference between either an experimental or theoretical distribution and a procrystal distribution, whereas the ELF is based on the ratio of the probability and the kinetic energy density. Despite this difference, it is interesting that the distributions are qualitatively similar.

Coesite, $\mathbf{S i O}_{2}$. The structure of this high pressure silica polymorph consists of a framework structure of corner sharing silicate $\mathrm{SiO}_{4}$ tetrahedra linked together by eight nonequivalent $\mathrm{SiO}$ bonded interactions that comprise five nonequivalent $\mathrm{SiOSi}$ angles that range in value between 137 and $180^{\circ}$. A generalized scattering factor modeling of the electron density distribution, ${ }^{17}$ using theoretical structure factor data generated with CRYSTAL98, ${ }^{18}$ was completed, and model $\Delta \rho$ and $L(r)$ isosurface maps were generated. ${ }^{4,19}$ The theoretical maps are in close correspondence with the experimental ones and display maxima along each of the $\mathrm{SiO}$ bond vectors and maxima on the reflex side of the bent angles as found for the $(\mathrm{HO})_{3} \mathrm{SiOSi}(\mathrm{OH})_{3}$ molecule. In contrast, the $\mathrm{O} 1$ atom involved in the straight angle is encircled in the experimental and theoretical maps by a ring torus with maxima located along each $\mathrm{SiO}$ bond vectors. The features of the level line contour map for the straight angle (Figure 2) are consistent with a ring torus distribution.

Dual $\eta(r)$ isosurface maps for the five nonequivalent SiOSi angles of coesite are compared with the theoretical model $\Delta \rho(r)$ maps in Figure 2 for the five nonequivalent bridging $\mathrm{O}$ atoms, $\mathrm{O} 1-\mathrm{O} 5$. The maps for the bent angles, involving $\mathrm{O} 2-\mathrm{O} 5$, are strikingly similar to local maxima displayed along each $\mathrm{SiO}$ bond vector and in the lone-pair reflex region of the bent angles. In the case of the straight angle involving O1, an isosurface deformation map displays a ring torus isosurface encircling the $\mathrm{O}$ atom, whereas banana shaped isosurfaces are displayed at the apices of the bent angles similar to that displayed by the $\eta(r)$ isosurface maps (see Figures 4 and 5 of ref 19). $L(r)$ isosurface maps for the angles were found to display similar features. ${ }^{19}$ Further, the $\eta(r)$ isosurface map generated for the bent $\mathrm{SiOSi}$ angle of the $(\mathrm{HO})_{3} \mathrm{SiOSi}(\mathrm{OH})_{3}$ molecule is strikingly similar to those generated for the bent angles in coesite. As the SiOSi angle in coesite decreases, the $L(r)$ maximum for the bridging $\mathrm{O}$ atoms is observed to increase in height, indicating that the nucleophilic character of the atom increases with decreasing angle. It is noteworthy that the $\mathrm{H}$ atoms in a $\mathrm{H}$-bearing coesite crystal have been determined to dock on the $\mathrm{O}$ atoms involved in the bent angles and to avoid $\mathrm{O} 1$, the $\mathrm{O}$ atom involved in the straight angle. ${ }^{20}$

Stishovite, $\mathbf{S i O}_{2}$. The structure of this very high pressure silica polymorph can also be described as a framework, but unlike coesite where each nonequivalent $\mathrm{O}$ atom is shared by two silicate tetrahedra, the structure consists of corner sharing silicate $\mathrm{SiO}_{6}$ octahedra where each $\mathrm{O}$ atom is shared by three equivalent $\mathrm{Si}$ atoms disposed at the corner of a triangle. The electron density distribution of stishovite has been modeled using conventional experimental single crystal $^{21,22}$ and synchrotron high energy single crystal X-ray diffraction data. ${ }^{6}$ A static $\Delta \rho$ map paralleling (110) and passing through the $\mathrm{O}$ atom and a triangle of three coordinating $\mathrm{Si}$ atoms is displayed in Figure (a)

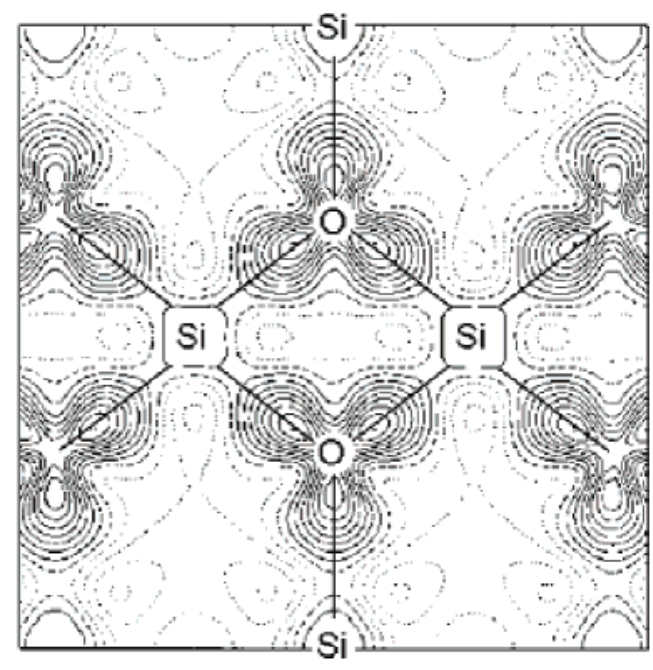

(b)

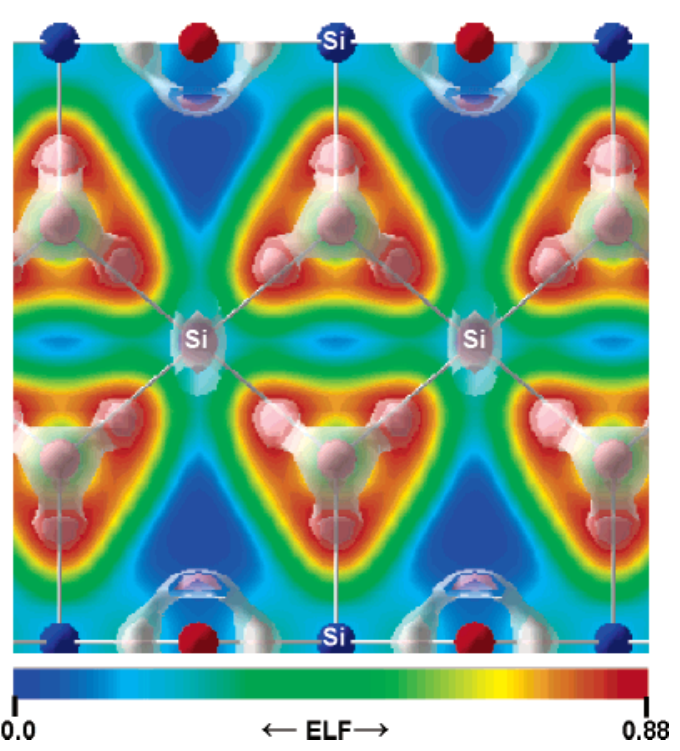

Figure 3. Static model $\Delta \rho(r)$ level line contour map (a) and combined $\eta(r)$ isosurface and color contour maps (b) for the (110) in stishovite. Each $\mathrm{Si}$ atom in the plane is bonded in the plane to four $\mathrm{O}$ atoms, and each $\mathrm{O}$ is bonded to three $\mathrm{Si}$ atoms. The contours are at $0.05 \AA^{-3}$, the dashed lines represent zero contour, the dotted lines represent negative contours, and the solid lines represent positive ones. Information describing the $\eta(r)$ isosurfaces is given in the legend of Figure 1. The color code for the ELF is defined in the bar. The red spheres represent oxygen atoms, and the blue ones represent silicon atoms.

3a. The map displays maxima along each $\mathrm{SiO}$ bond vector at a distance of $\sim 0.70 \AA$ from the $\mathrm{O}$ atom (Figure 3a, ref 6 ). An $\eta(r)$ color contour map of the plane is displayed in Figure $3 \mathrm{~b}$. As observed in the level line contour map, each $\mathrm{O}$ atom is surrounded by a triangular array of color contours which increase from light green to orange. The shapes of the isosurfaces conform with the contours displayed in the figure with one of the maxima directed along a $\mathrm{SiO}$ bond vector and the other two each displaced to the outside of the SiOSi angle $\sim 0.1 \AA$ displayed along each of the three $\mathrm{SiO}$ bond vectors. The $\Delta \rho$ maxima each lie along the $\mathrm{SiO}$ vectors at $\sim 0.45 \AA$, on average, from the $\mathrm{O}$ atoms. One of the $\eta(r)$ maxima lies along the vector, while the other two are displaced off the vectors $\sim 0.1 \AA$ on the exterior side of the SiOSi angle. Nonetheless, the features displayed by the $\eta(r)$ distribution correspond closely to those displayed by the $\Delta \rho$ distribution. The pink isosurface displayed above the $\mathrm{O}$ atom at the center of the triangle has been ascribed to a lone-pair domain, a feature displayed in experimental $\Delta \rho(r)$ maps recorded by Spackman et al. ${ }^{21}$ and Kirfel et al. ${ }^{6}$ This 
(a)

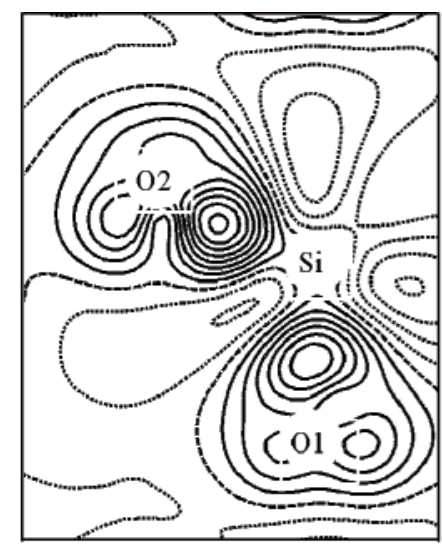

(b)
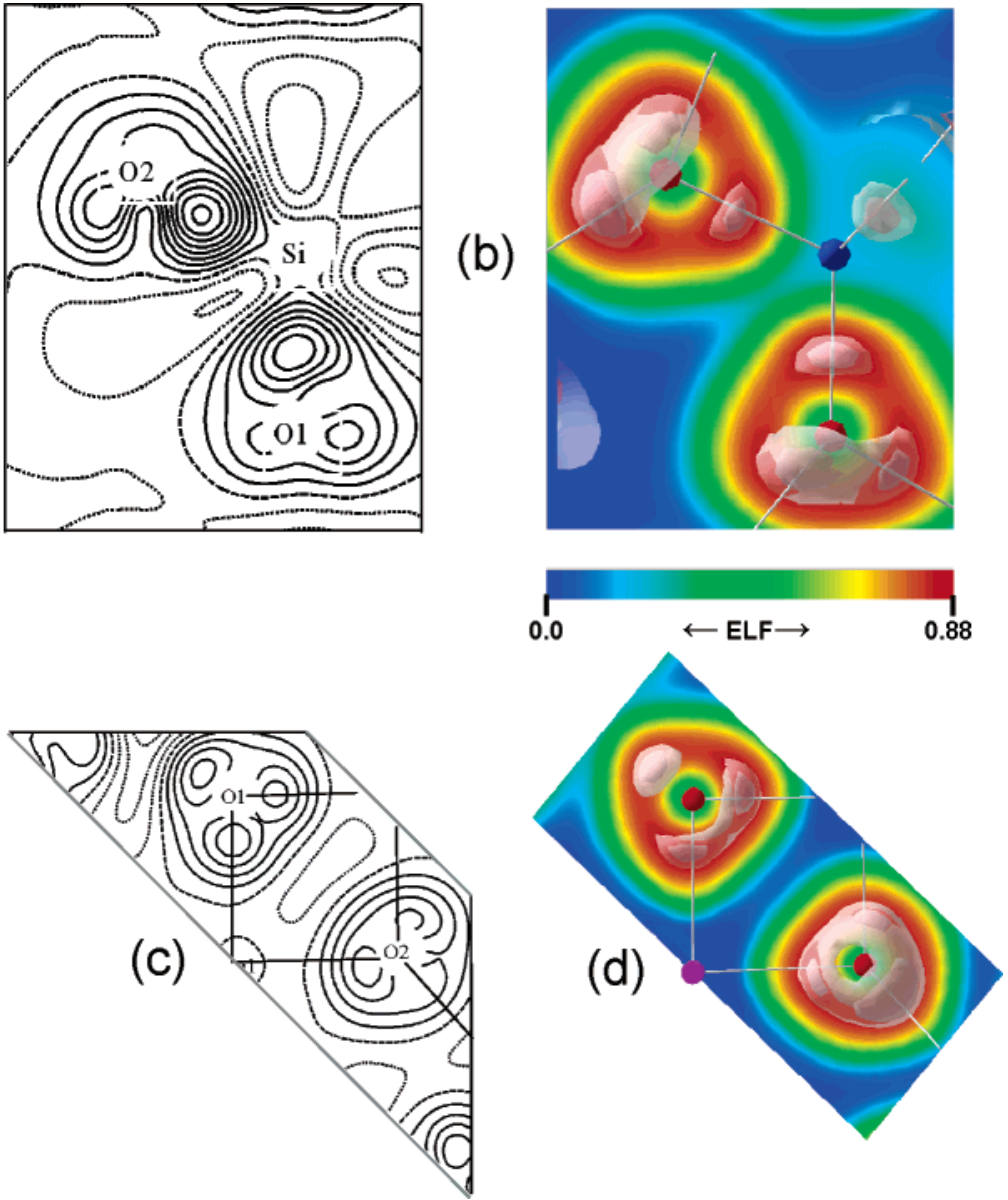

Figure 4. Static model $\Delta \rho(r)$ level line contour maps for a plane containing the $\mathrm{O} 2 \mathrm{SiO} 1$ atoms of the silicate tetrahedron (a) and the $\mathrm{O} 1 \mathrm{Mg} 1 \mathrm{O} 2$ atoms of the $\mathrm{Mg} 1 \mathrm{O}_{6}$ octahedron (c) in forsterite and combined $\eta(r)$ and color contour maps for the same planes (b and d, respectively). See the information describing the $\eta(r)$ isosurfaces and color contours given in the legend of Figure 1. The red spheres represent oxygen atoms, the blue ones represent silicon atoms, and the magenta ones represent magnesium atoms.

domain is displayed above (below) each $\mathrm{O}$ atom at the bottom (top) of Figure $3 \mathrm{~b}$ where the triangle is viewed end on. As asserted by Gibbs et al., ${ }^{23}$ this lone-pair domain plays a role in governing the position adopted by the $\mathrm{H}$ atom in $\mathrm{H}$,Al-bearing stishovite.

Forsterite, $\mathrm{Mg}_{2} \mathrm{SiO}_{4}$. The structure of this orthosilicate can be described as a distorted close packed array of $\mathrm{O}$ atoms in which one-half of the available octahedral voids are occupied by $\mathrm{Mg}$ atoms and one-eighth of the available tetrahedral voids are occupied by $\mathrm{Si}$ such that each $\mathrm{O}$ is bonded to three $\mathrm{Mg}$ atoms and a $\mathrm{Si}$ atom. The crystal structure was recently determined using essentially extinction and adsorption free high energy synchrotron single crystal X-ray diffraction data. ${ }^{5} \mathrm{~A}$ generalized scattering factor modeling of the electron density resulted in a set of bond critical point properties that agree with those generated with first principles quantum chemical calculations within $\sim 5 \%$. This agreement not only showed that the modeling is adequate but also showed that the model experimental and theoretical electron density is relatively accurate. Static model experimental deformation contour maps of the distribution for the $\mathrm{O} 1 \mathrm{SiO} 2$ plane of the silicate $\mathrm{SiO}_{4}$ tetrahedral oxyanion and the $\mathrm{O} 1 \mathrm{O} 2$ edge shared in common between two $\mathrm{Mg}_{10} \mathrm{O}_{6}$ octahedral oxyanions are displayed in Figure $4 \mathrm{a}$ and c. ${ }^{5}$ Well developed maxima are displayed in the contour maps along the $\mathrm{SiO}$ bond vectors together with satellite maxima in the vicinity of the $\mathrm{O} 1$ and $\mathrm{O} 2$ atoms. Figure $4 \mathrm{~b}$ displays an $\eta(r)$ color contour map for the $\mathrm{O} 1 \mathrm{SiO} 2$ plane with a superimposed $\eta(r)$ dual isosurface. As observed in the experimental map, the $\eta(r)$ hemispherical isosurfaces are displayed along the $\mathrm{SiO} 1$ and $\mathrm{SiO} 2$ bond vectors and toroidal isosurfaces are displayed in the vicinity of two $\mathrm{O}$ atoms. The latter enclose maxima displayed along the $\mathrm{MgO}$ bond vectors that radiate from $\mathrm{O} 1$ and $\mathrm{O} 2$, and the isosurface coming out of the plane encloses a maximum along the $\mathrm{SiO} 3$ bond vector not displayed in the contour map. Figure $4 \mathrm{c}$ is a level line $\Delta \rho$ contour map of the edge shared in common between two $\mathrm{Mg}_{10} \mathrm{O}_{6}$ octahedra. The $\mathrm{O} 1$ atom is coordinated by three well-defined maxima, two of which are displayed along $\mathrm{Mg} 1 \mathrm{O} 1$ bond vectors and the third along a SiO1 vector. Two well-defined maxima together with a less defined one coordinate $\mathrm{O} 2$. The well-defined ones are displayed along $\mathrm{Mg} 1 \mathrm{O} 2$ displayed along the $\mathrm{Mg} 1 \mathrm{O} 2$ vectors and $\mathrm{MgO} 2$ bond vectors. The less defined maximum lies along a $\mathrm{Mg} 2 \mathrm{O} 2$ vector that is directed out of the plane of the shared edge. The isosurfaces displayed in the $\eta(r)$ color contour isosurface map (Figure 4d) of the shared edge show a close correspondence to those displayed in the contour map in both number and arrangement. However, the heights of the $\Delta \rho(r)$ maxima along the $\mathrm{SiO}$ bond vectors of the silicate group are substantially higher than those in the nonbonded region. On the other hand, the reverse is true in the $\eta(r)$ isosurface maps where the isosurfaces along the $\mathrm{SiO}$ bond vector are smaller than those displayed in the nonbonded regions.

Magnesite, $\mathrm{MgCO}_{3}$. The $\mathrm{O}$ atoms in this carbonate can be viewed as roughly hexagonally close packed with the $\mathrm{Mg}$ atoms filling one-third of the available octahedral voids with $\mathrm{C}$ coordinated by three $\mathrm{O}$ atoms in the individual monolayers of the structure such that each $\mathrm{O}$ atom is bonded to two $\mathrm{Mg}$ atoms and a $\mathrm{C}$ atom. Using theoretical structure factors generated with 
(a)

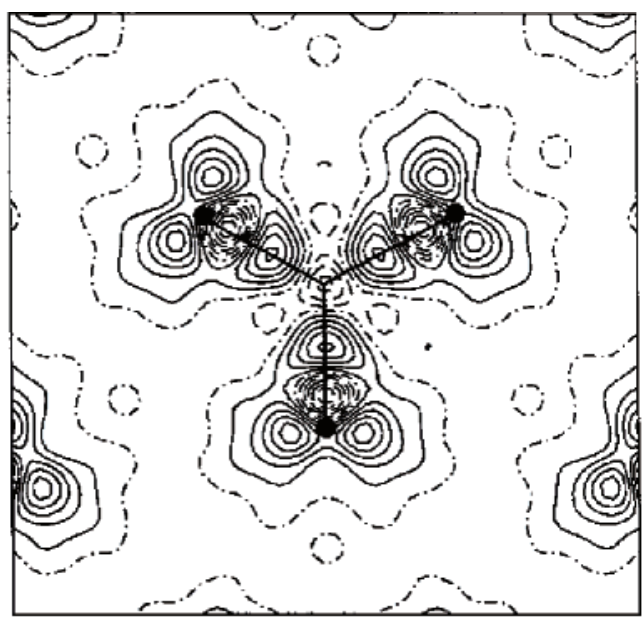

(b)

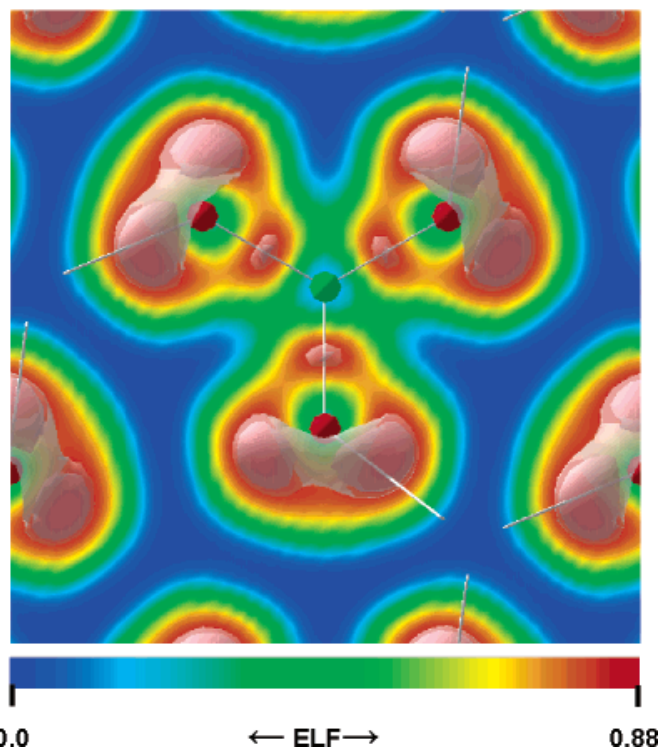

Figure 5. Static model $\Delta \rho(r)$ level line contour map for the carbonate group in magnesite generated with theoretical structure factors (a) and combined $\eta(r)$ isosurface and color contour maps (b). The solid, dashed, and dot-dashed level contour lines refer to positive, negative, and zero values, respectively. The interval between the level contour lines is $0.1 \mathrm{e} \AA^{-3}$. Information describing the $\eta(r)$ isosurfaces and color contours is given in the legend of Figure 1. The red spheres represent oxygen atoms, and the green one represents a carbon atom.

quantum mechanical periodic Hartree-Fock methods, Catti and Pavese ${ }^{24}$ calculated a deformation map for the $\mathrm{CO}_{3}$ molecular group displayed in Figure 5a. Well-defined maxima are displayed along each $\mathrm{CO}$ bond vector. In addition, each $\mathrm{O}$ atom is coordinated by two additional maxima that reside in the nonbonded domains of the atom. An $\eta(r)$ color and isosurface mapping for the plane defined by the $\mathrm{CO}_{3}$ molecular groups displays strikingly similar features to those displayed in the $\Delta \rho(r)$ map. The maps differ in that the maxima along the CO bond vectors in the $\Delta \rho(r)$ maps are similar in magnitude to those in the nonbonded region, whereas the maxima displayed along the $\mathrm{CO}$ vectors in the $\eta(r)$ maps are substantially smaller than those in the nonbonded region. As observed for forsterite, the maxima in the nonbonded region likewise tend to be directed toward the $\mathrm{Mg}$ atoms, suggesting covalent bonded interactions between the $\mathrm{Mg}$ atoms and $\mathrm{O}$ atoms of the $\mathrm{MgO}_{6}$ octahedra. In a study of $\eta(r)$ maps for a number of silicates, maxima in the lone-pair region for a number of silicates were also observed to be directed toward the nontetrahedral atoms. ${ }^{3}$ As observed for the silicate group in forsterite, the $\eta(r)$ isosurfaces displayed along the $\mathrm{CO}$ bond vectors of the $\mathrm{CO}_{3}$ molecular group are substantially smaller than those displayed in the nonbonded regions (Figure $5 b$ ). In contrast, the heights of the $\Delta \rho(r)$ maxima displayed in the bonded and nonbonded regions of the carbonate group are virtually identical (Figure 5a).

\section{Concluding Remarks}

The arrangement and number of the domains displayed by $\eta(r)$ and $\Delta \rho(r)$ maps generated in this study are strikingly similar as observed by Bader et al. ${ }^{1}$ for the $\eta(r)$ and $L(r)$ maps observed for a number of molecules. This not only suggests that the $\eta(r)$ and $\Delta \rho(r)$ fields are homeomorphically related in terms of the number and arrangement of the domains of lone-pair electrons but also suggests that they are also related to the $L(r)$ field. This seems to be the case for the several cases examined in this study where the number of domains and their arrangement displayed in $\Delta \rho(r), L(r)$, and $\eta(r)$ maps are similar. ${ }^{19}$ However, $\Delta \rho(r)$ is believed to be superior to $\eta(r)$ in the prediction of the location of the localized domains of electron density given its experimental basis.

Acknowledgment. The National Science Foundation and The U.S. Department of Energy are thanked for supporting this study in part with grants EAR-0229472 (N.L.R., G.V.G.), DEFG02-03ER15389 (J. D. Rimstidt, G.V.G.), and DE-FG0297ER14751 (D.F.C.). We thank Richard Godbee for his assistance in generating Figure 5 and an anonymous reviewer for suggestions that significantly improved the manuscript.

\section{References and Notes}

(1) Bader, R. F. W.; Johnson, S.; Tang, T. H.; Popelier, P. L. A. J. Phys. Chem. A 1996, 100, 15398-15415.

(2) Bader, R. F. W.; Gillespie, R. J.; MacDougall, P. J. J. Am. Chem. Soc. 1988, 110, 7329-7336.

(3) Gibbs, G. V.; Cox, D. F.; Ross, N. L.; Crawford, T. D.; Burt, J. B.; Rosso, K. M. Phys. Chem. Miner. 2005, 32, 208-221.

(4) Gibbs, G. V.; Whitten, E. W.; Spackman, M. A.; Stimpfl, M.; Downs, R. T.; Carducci, M. D. J. Phys. Chem. B 2003, 107, 12996-13006.

(5) Kirfel, A.; Lippmann, T.; Blaha, P.; Schwarz, K.; Cox, D. F.; Rosso, K. M.; Gibbs, G. V. Phys. Chem. Miner. 2005, 32, 301-313.

(6) Kirfel, A.; Krane, H. G.; Blaha, P.; Schwarz, K.; Lippmann, T. Acta Crystallogr., Sect. A 2001, 57, 663-677.

(7) Kresse, G.; Hafner, J. Phys. Rev. B 1993, 47, 558-561.

(8) Kresse, G.; Hafner, J. Phys. Rev. B 1994, 49, 14251-14269.

(9) Kresse, G.; Furthmüller, J. Phys. Rev. B 1996, 54, 11169-11186.

(10) Vanderbilt, D. Phys. Rev. B 1990, 41, 7892-7895.

(11) Monkhorst, H. J.; Pack, J. D. Phys. Rev. B 1976, 13, 5188-5192.

(12) Terriberry, T. B.; Cox, D. F.; Bowman, D. A. Comput. Chem. 2002 $26,313-319$

(13) Burdett, J. K.; McCormick, T. A. J. Phys. Chem. A 1998, 102, $6366-6372$

(14) Becke, A. D.; Edgecombe, K. E. J. Phys. Chem. 1990, 92, 53975403.

(15) Gibbs, G. V.; Cox, D. F.; Crawford, T. D.; Boisen, M. B.; Lim, M. Phys. Chem. Miner. 2002, 29, 307-318.

(16) Coppens, P. X-ray Charge Densities and Chemical Bonding; Oxford University Press: New York, 1997.

(17) Stewart, R. F. Acta Crystallogr., Sect. A 1976, 32, 565-574.

(18) Saunders, V. R.; Dovesi, R.; Roetti, C.; Causá, M.; Harrison, N. M.; Orlando, R.; Aprá, E. CRYSTAL98 User's Manual; University of Torino: Torino, Italy, 1998; pp 1-170.

(19) Gibbs, G. V.; Cox, D. F.; Boisen, M. B.; Downs, R. T.; Ross, N. L. Phys. Chem. Miner. 2003, 30, 305-316.

(20) Koch-Müller, M.; Fei, Y.; Hauri, E.; Liu, Z. Phys. Chem. Miner. 2001, 28, 693-705

(21) Hill, R. J.; Newton, M. D.; Gibbs, G. V. J. Solid State Chem. 1983 , $47,185-200$.

(22) Spackman, M. A.; Hill, R. J.; Gibbs, G. V. Phys. Chem. Miner. 1987, 14, 139-150.

(23) Gibbs, G. V.; Cox, D. F.; Ross, N. L. Phys. Chem. Miner. 2004 $31,232-239$.

(24) Catti, M.; Pavese, A. Acta Crystallogr., Sect. A 1996, 52, 413418. 